Marin H. Kollef

\title{
What can be expected from antimicrobial de-escalation in the critically ill?
}

Received: 3 November 2013

Accepted: 4 November 2013

Published online: 19 November 2013

(C) Springer-Verlag Berlin Heidelberg and ESICM 2013

M. H. Kollef (『)

Division of Pulmonary and Critical Care Medicine,

Washington University School of Medicine, 660 South Euclid

Avenue, Campus Box 8052, St. Louis, MO 63110, USA

e-mail: mkollef@dom.wustl.edu

Tel.: +1-314-4548764

Fax: +1-314-4545571

Antimicrobial de-escalation is a clinical approach to empirical antibiotic treatment of serious infections that attempts to balance the need for appropriate initial therapy with the need to limit unnecessary antimicrobial exposure in order to curtail the emergence of resistance [1]. When risk factors for antibiotic resistance are identified in patients with a serious infection, broad-spectrum antimicrobials should be prescribed. A de-escalation approach usually requires initial combination antimicrobial treatment targeting resistant non-fermenting gramnegative bacilli (NFGNB) (Pseudomonas aeruginosa, Acintobacter species) and methicillin-resistant Staphylococcus aureus [2]. However, depending on clinical presentation, patient risk factors, and local epidemiology, other pathogens such as Candida species and Clostridium difficile, especially when diarrhea is present, may also need to be covered. Once the microbiologic results are available and the patient's clinical response is observed, the antibiotic regimen can be narrowed on the basis of the susceptibilities of the identified pathogens.

In addition to narrowing antibiotic regimens, de-escalation implies that the shortest course of antibiotic treatment should be prescribed that adequately treats the underlying infection. For uncomplicated nosocomial pneumonia, this may be as little as 7 days of therapy [3]. This is an important aspect of de-escalation, as the duration of antibiotic treatment appears to be one of the most important, if not the most important, determinant for the emergence of antimicrobial resistance in hospitalized patients [4]. Moreover, in order to achieve optimal outcomes, including reductions in mortality and shorter courses of antibiotic administration aimed at minimizing the pressure for resistance to emerge, the initial antibiotic regimen should be administered in a timely manner and appropriate for the underlying infection (i.e., active against the pathogen associated with infection based on in vitro susceptibility testing) [4-6]. Although the concept of antimicrobial de-escalation seems to make intuitive sense, clinicians should ask themselves what the realistic expectations of such a strategy are.

Intensivists should expect that a de-escalation approach to antimicrobial therapy in critically ill patients will optimize patient outcomes. Our local experience, as well as that of other groups, bears this out in demonstrating that the administration of appropriate initial antibiotic therapy is associated with improved survival and shorter hospital stays [2, 5-7]. Recently, Garnacho-Montero et al. [8] evaluated 628 patients with severe sepsis or septic shock at ICU admission who were treated empirically with broad-spectrum antibiotics. Antibiotic therapy was guided by written protocols advocating for de-escalation therapy once the microbiological results became available (day of culture results), although this decision was ultimately the responsibility of the physician in charge of the patient. By multivariate analysis, factors independently associated with in-hospital mortality were septic shock, SOFA score on the day of culture results, and inappropriate empirical antimicrobial therapy, whereas de-escalation of antimicrobial therapy was found to be a protective factor for hospital survival. Additionally, among patients receiving appropriate therapy the only factor independently associated with 
mortality was SOFA score on the day of culture results, whereas de-escalation therapy was again found to be a protective factor. These investigators found that 57 of 628 $(9.1 \%)$ patients received inappropriate empiric therapy and 246 of $628(39.2 \%)$ patients had no change in their empiric antibiotic regimens, indicating further opportunity to improve their de-escalation practice.

Several strategies have been employed to optimize the use of antimicrobial de-escalation in critically ill patients. Rello et al. [9] conducted a prospective study utilizing a protocol to guide de-escalation of therapy in patients with ventilator-associated pneumonia (VAP). Changes in empiric antibiotic therapy occurred in $56.2 \%$, including de-escalation (the most frequent cause) in $31.4 \%$ (increasing to $38 \%$ if isolates were sensitive). De-escalation was lower $(p<0.05)$ in the presence of NFGNB $(2.7$ vs. $49.3 \%$ ) and in the presence of late-onset pneumonia $(12.5$ vs. $40.7 \%)$. When the pathogen remained unknown, half of the patients died and de-escalation was not performed. Ibrahim et al. [2] conducted a before-after trial of standard therapy versus a de-escalation guideline for the treatment of VAP. De-escalation included both narrowing the spectrum of therapy on the basis of microbiology results and shortening the duration of antibiotic therapy on the basis of the patient's clinical response. These investigators found that the initial administration of appropriate antimicrobial treatment was statistically greater during the afterperiod compared with the before-period (48.0 vs. $94.2 \%$, $p<0.001)$ and that the duration of antimicrobial treatment was statistically shorter during the after-period $(14.8 \pm 8.1$ vs. $8.6 \pm 5.1$ days, $p<0.001$ ). Second episodes of VAP also occurred less often among patients in the after-period ( 24.0 vs. $7.7 \%, p=0.030$ ).

Computer decision support systems have also been employed to facilitate de-escalation practices in the ICU setting. Thursky et al. [10] employed a real-time microbiology browser and computerized decision support system for isolate-directed antibiotic prescription. They found a significant reduction in the proportion of patients prescribed carbapenems, third-generation cephalosporins, and vancomycin after adjustment for risk factors including Apache II score, suspected infection, positive microbiology, intubation, and length of stay. The decision support tool was associated with a $10.5 \%$ reduction in both total antibiotic utilization (166-149 defined daily doses/100 ICU bed days) and the highest volume broad-spectrum antibiotics. Our own hospital is developing an automated decision-support system with real-time access to patients'

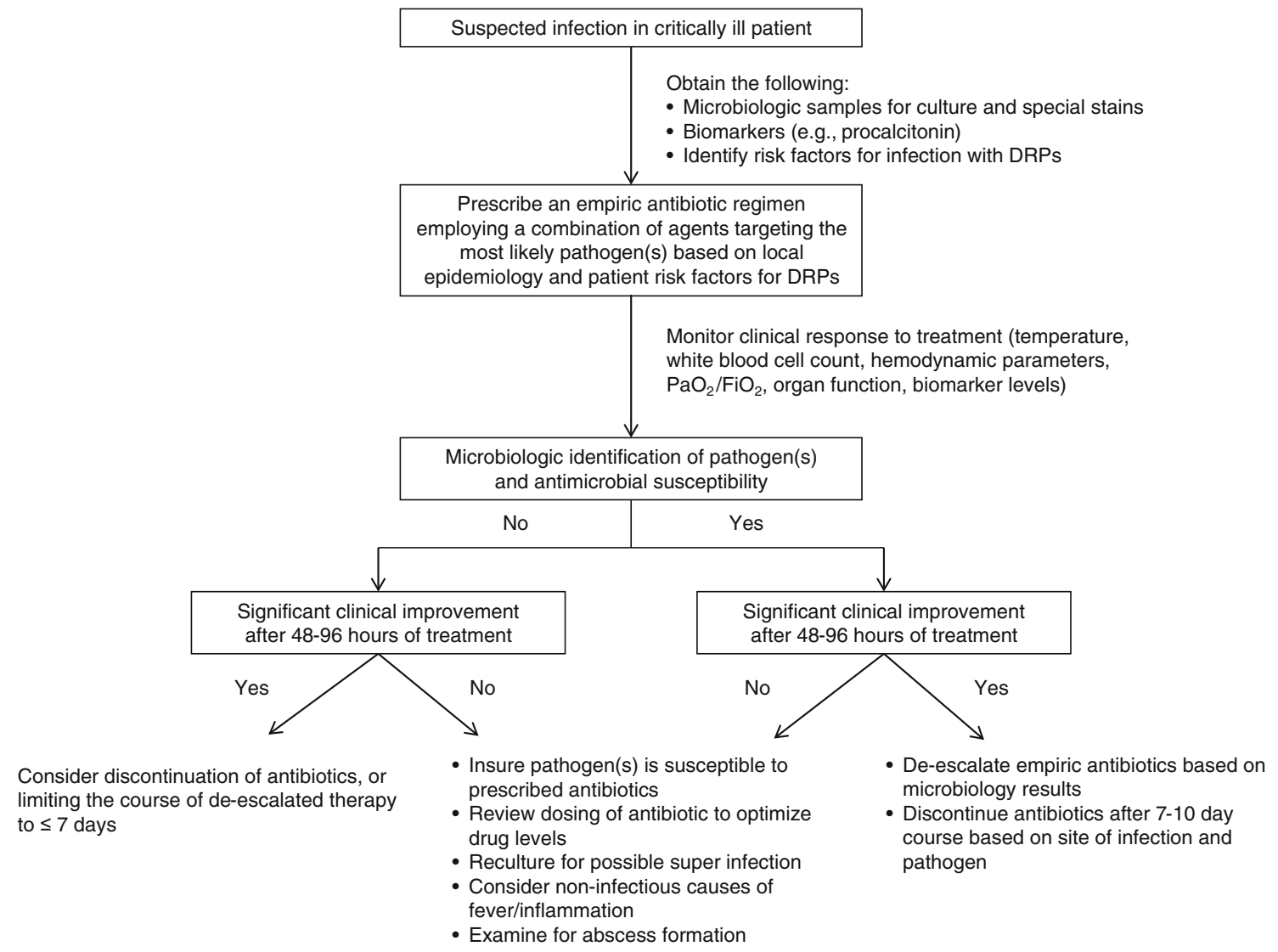

Fig. 1 Schematic outlining a practice of antimicrobial de-escalation. $\mathrm{FiO}_{2}$ inspired oxygen fraction, $\mathrm{PaO}_{2}$ partial pressure (or tension) of arterial oxygen, DRPs drug-resistant pathogens 
prior antibiotic exposures and microbiologic results, including those from prior hospitalizations at outside institutions, in order to refine our current empiric antibiotic practices and assist in the performance of de-escalation.

Knowledge of patient risk factors for the presence of infection with antibiotic-resistant pathogens should be a routine part of antibiotic decision-making and can be used in a de-escalation algorithm. For example, communityacquired pneumonia (CAP) drug-resistant pathogens (DRPs) are more commonly found in patients with healthcare-associated risk factors. Shindo et al. [11] demonstrated that independent risk factors for DRPs in both patients diagnosed with CAP and healthcare-associated pneumonia (HCAP) included prior hospitalization, immunosuppression, previous antibiotic use, use of gastric acid-suppressive therapy, tube feeding, and nonambulatory status. These are similar to independent risk factors identified in a clinical score for assessing the risk of resistant pathogens in patients with pneumonia presenting to the emergency department [12]. Identification of the presence or absence of such risk factors at the time of antibiotic decision-making can obviate the need for broad-spectrum therapy in patients without risk factors for DRPs and avoid having to de-escalate therapy, especially in culture-negative patients. Moreover, biomarkers are increasingly employed to modify empiric antibiotic therapy, including in critically ill patients. Available evidence suggests that biomarker-prompted de-escalation of empiric therapy can be safely applied, although additional trials are needed to confirm this approach [13].

In summary, antibiotic de-escalation should be a routine part of antimicrobial stewardship as it is applied in the ICU. Successful implementation of de-escalation strategies will require a multidisciplinary approach with dedicated efforts and monitoring to insure adherence to its guidance principles [14]. Given the increasing presence of antibiotic-resistant pathogens as a cause of infection in critically ill patients, a practice of de-escalation appears to be the only available practical strategy allowing clinicians to balance the need for empiric appropriate therapy while minimizing the unnecessary use of antibiotics. Certainly, the future development of rapid methods for microbe detection and antimicrobial susceptibility testing will allow for more timely and directed therapy for critically ill patients with serious infections [15]. Until that time, those of us who treat patients in the ICU setting should champion antibiotic de-escalation as a tool to manage our use of antimicrobial agents (Fig. 1).

\section{References}

1. Kollef MH, Micek ST (2005) Strategies to prevent antimicrobial resistance in the intensive care unit. Crit Care Med 33:1845-1853

2. Ibrahim EH, Ward S, Sherman G, Schaiff R, Fraser VJ, Kollef MH (2001) Experience with a clinical guideline for the treatment of ventilator-associated pneumonia. Crit Care Med 29:1109-1115

3. Chastre J, Wolff M, Fagon JY, Chevret S, Thomas F, Wermert D, Clementi E, Gonzalez J, Jusserand D, Asfar P, Perrin D, Fieux F, Aubas S, PneumA Trial Group (2003) Comparison of 15 vs. 8 days of antibiotic therapy for ventilator-associated pneumonia in adults: a randomized trial. JAMA 290:2588-2598

4. Dennesen PJ, van der Ven AJ, Kessels AG, Ramsay G, Bonten MJ (2001) Resolution of infectious parameters after antimicrobial therapy in patients with ventilator-associated pneumonia. Am J Respir Crit Care Med 163:1371-1375

5. Kollef MH, Sherman G, Ward S, Fraser VJ (1999) Inadequate antimicrobial treatment of infections: a risk factor for hospital mortality among critically ill patients. Chest 115:462-474
6. Ferrer R, Artigas A, Suarez D, Palencia E, Levy MM, Arenzana A, Pérez XL, Sirvent JM (2009) Effectiveness of treatments for severe sepsis: a prospective, multicenter, observational study. Am J Respir Crit Care Med 180:861-866

7. Shorr AF, Micek ST, Welch EC, Doherty JA, Reichley RM, Kollef MH (2011) Inappropriate antibiotic therapy in gram-negative sepsis increases hospital length of stay. Crit Care Med 39:46-51

8. Garnacho-Montero J, GutiérrezPizarraya A, Escoresca-Ortega A, Corcia-Palomo Y, Fernández-Delgado E, Herrera-Melero I, Ortiz-Leyba C, Márquez-Vácaro JA (2013) Deescalation of empirical therapy is associated with lower mortality in patients with severe sepsis and septic shock. Intensive Care Med. doi: 10.1007/s00134-013-3077-7

9. Rello J, Vidaur L, Sandiumenge A, Rodríguez A, Gualis B, Boque C, Diaz E (2004) De-escalation therapy in ventilator-associated pneumonia. Crit Care Med 32:2183-2190
10. Thursky KA, Buising KL, Bak N, Macgregor L, Street AC, Macintyre CR, Presneill JJ, Cade JF, Brown GV (2006) Reduction of broad-spectrum antibiotic use with computerized decision support in an intensive care unit. Int J Qual Health Care 18:224-231

11. Shindo Y, Ito R, Kobayashi D, Ando M, Ichikawa M, Shiraki A, Goto Y, Fukui Y, Iwaki M, Okumura J, Yamaguchi I, Yagi T, Tanikawa Y, Sugino Y, Shindoh J, Ogasawara T, Nomura F, Saka H, Yamamoto M, Taniguchi H, Suzuki R, Saito H, Kawamura T, Hasegawa Y (2013) Risk factors for drug-resistant pathogens in communityacquired and healthcare-associated pneumonia. Am J Respir Crit Care Med 188:985-995

12. Shorr AF, Zilberberg MD, Reichley R, Kan J, Hoban A, Hoffman J, Micek ST, Kollef MH (2012) Validation of a clinical score for assessing the risk of resistant pathogens in patients with pneumonia presenting to the emergency department. Clin Infect Dis 54:193-198 
13. Schuetz P, Mueller B (2013)

Biomarker-guided de-escalation of empirical therapy is associated with lower risk for adverse outcomes. Intensive Care Med. doi: 10.1007/s00134-013-3139-x
14. Micek ST, Ward S, Fraser VJ, Kolle MH (2004) A randomized controlled trial of an antibiotic discontinuation policy for clinically suspected ventilator-associated pneumonia. Chest 125:1791-1799
15. Pulido MR, García-Quintanilla M, Martín-Peña R, Cisneros JM, McConnell MJ (2013) Progress on the development of rapid methods for antimicrobial susceptibility testing. J Antimicrob Chemother 68:2710-2717 